\title{
The fibrodysplasia ossificans progressiva R206H ACVR1 mutation activates BMP-independent chondrogenesis and zebrafish embryo ventralization
}

\author{
Qi Shen,1,2 Shawn C. Little, ${ }^{3}$ Meiqi Xu,1,2 Julia Haupt,4,5,6 Cindy Ast, ${ }^{5}$ Takenobu Katagiri,7
} Stefan Mundlos, 4,5,6 Petra Seemann, ${ }^{5,6}$ Frederick S. Kaplan, ${ }^{1,2,8}$

Mary C. Mullins, ${ }^{3}$ and Eileen M. Shore ${ }^{1,2,9}$

\begin{abstract}
1Department of Orthopaedic Surgery, ${ }^{2}$ Center for Research in FOP and Related Disorders, and ${ }^{3}$ Department of Cell and Developmental Biology, University of Pennsylvania School of Medicine, Philadelphia, Pennsylvania, USA. ${ }^{4}$ nstitut für Medizinische Genetik, Charité-Universitätsmedizin Berlin, Berlin, Germany. ${ }^{5}$ Max-Planck-Institut für Molekulare Genetik, Research Group Development and Disease, Berlin, Germany.

${ }^{6}$ Berlin Brandenburg Center for Regenerative Therapies (BCRT), Charité-Universitätsmedizin Berlin, Berlin, Germany. ${ }^{7}$ Division of Pathophysiology, Research Center for Genomic Medicine, Saitama Medical University, Yamane, Hidaka-shi, Saitama, Japan. ${ }^{8}$ Department of Medicine and PDepartment of Genetics, University of Pennsylvania School of Medicine, Philadelphia, Pennsylvania, USA.
\end{abstract}

\begin{abstract}
Patients with classic fibrodysplasia ossificans progressiva, a disorder characterized by extensive extraskeletal endochondral bone formation, share a recurrent mutation $(\mathrm{R} 206 \mathrm{H})$ within the glycine/serine-rich domain of ACVR1/ALK2, a bone morphogenetic protein type I receptor. Through a series of in vitro assays using several mammalian cell lines and chick limb bud micromass cultures, we determined that mutant R206H ACVR1 activated BMP signaling in the absence of BMP ligand and mediated BMP-independent chondrogenesis that was enhanced by BMP. We further investigated the interaction of mutant R206H ACVR1 with FKBP1A, a glycine/serine domain-binding protein that prevents leaky BMP type I receptor activation in the absence of ligand. The mutant protein exhibited reduced binding to FKBP1A in COS-7 simian kidney cell line assays, suggesting that increased BMP pathway activity in COS-7 cells with R206H ACVR1 is due, at least in part, to decreased binding of this inhibitory factor. Consistent with these findings, in vivo analyses of zebrafish embryos showed BMP-independent hyperactivation of BMP signaling in response to the $\mathrm{R} 206 \mathrm{H}$ mutant, resulting in increased embryonic ventralization. These data support the conclusion that the mutant $\mathrm{R} 206 \mathrm{H}$ ACVR1 receptor in FOP patients is an activating mutation that induces BMP signaling in a BMP-independent and BMP-responsive manner to promote chondrogenesis, consistent with the ectopic endochondral bone formation in these patients.
\end{abstract}

\section{Introduction}

Fibrodysplasia ossificans progressiva (FOP; MIM 135100), a rare genetic disorder of progressive extraskeletal (heterotopic) ossification, is the most severe form of human heterotopic ossification known and results in profoundly decreased mobility of affected individuals (1). Patients with classic FOP have congenital malformation of the great toes and develop progressive heterotopic ossification within soft connective tissues in characteristic anatomic patterns $(2,3)$. Ectopic bone formation in FOP occurs through an endochondral pathway in which cartilage forms initially at the lesional site and is subsequently replaced by bone $(4,5)$. The genetic mutation in FOP is therefore a likely key regulator of cartilage and bone formation.

The gene mutation for patients with the classic FOP clinical phenotype was mapped to chromosome 2q23-24, and mutations were identified in activin A receptor, type I (ACVR1; also known as $A L K 2$ ), which encodes a bone morphogenetic protein (BMP) type I receptor (6). ACVR1 is expressed in several tissues, including cartilage and skeletal muscle, consistent with both the congenital skeletal malformations and the sites of postnatal endochondral het-

Conflict of interest: The authors have declared that no conflict of interest exists. Citation for this article: J. Clin. Invest. 119:3462-3472 (2009). doi:10.1172/JCI37412. erotopic ossification in FOP. Previous reports demonstrated that constitutive activation of ACVR1 induces alkaline phosphatase (ALP) activity, expands cartilage elements, and induces joint fusions, all of which are associated with enhanced chondrogenesis and/or dysregulation of the BMP signaling pathway (7-9).

BMPs, members of the TGF- $\beta$ superfamily, were identified initially as a family of proteins that could induce ectopic bone formation through an endochondral process (10-12). BMPs are critical signaling proteins, not only for bone and cartilage formation, but also in many stages of vertebrate development (13). During in vivo vertebrate development, BMP signaling is first required in early embryogenesis and is involved in the patterning of the tissues along the dorsal-ventral axis (14).

BMPs are extracellular ligands that exert their effects by binding to complexes of type I and type II serine/threonine kinase BMP receptors that are located at the cell membrane (15-19). Four type I receptors (ACVR1 [ALK2], BMPR1A [ALK3], BMPR1B [ALK6], and ACVRL1 [ALK1]) can mediate BMP signal transduction. Three type II receptors have been identified: BMPR2 (BMPRII), ACVR2A (ActRII), and ACVR2B (ActR-IIB). In the absence of BMP binding, the FK506-binding protein 1A (FKBP1A protein, also called FKBP12) binds the glycine-serine (GS) domain of BMP type I receptors to inhibit internalization of the receptor and down- 
A
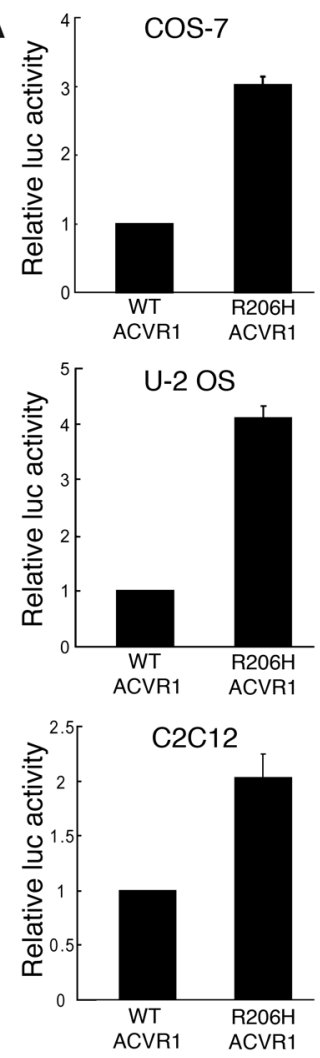

B
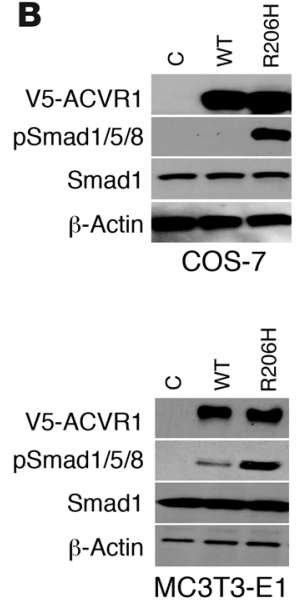

Figure 1

Mutant ACVR1 (c617A; R206H) activates the BMP signaling pathway. (A) Activation of ID1 transcription. COS-7 cells were cotransfected with an ID1 promoter luciferase reporter construct $(-985 /+94)$ and either human wild-type ACVR1 or mutant ACVR1 (R206H) expression vectors. Similar assays used U-2 OS and $\mathrm{C} 2 \mathrm{C} 12$ cells. Data represent mean \pm SEM $(n>3)$. (B) Induction of Smad1/5/8 phosphorylation. COS-7 (top) or MC3T3-E1 cells (bottom) were transfected with V5-tagged wild-type ACVR1 or c617A mutant ACVR1 (R206H) constructs or with the empty vector control (C). Immunoblot analysis for phosphorylated Smad1/5/8 showed induction of Smad1/5/8 phosphorylation without BMP treatment in cells expressing the mutant but not wild-type ACVR1 or empty vector constructs. Total Smad1 protein is shown. V5 antibody was used to detect the expression of the V5tagged ACVR1 proteins. $\beta$-Actin was detected as a loading control. Data are representative of 3 individual experiments.

tion of BMP activation in vivo. Furthermore, in cell differentiation assays, the ACVR1 mutant receptor induces enhanced chondrogenesis that is both BMP independent and BMP responsive. These data provide strong evidence suggesting that the mutant $\mathrm{R} 206 \mathrm{H}$ ACVR1 receptor induces dysregulation of BMP signaling to promote heterotopic endochondral ossification in FOP patients.

\section{Results}

The FOP mutant ACVR1 receptor (c.617A; R206H) activates BMP-independent signaling of the BMP pathway. To examine the functional effects of the ACVR1 c.617G $\rightarrow$ A; R206H mutation that occurs in classic FOP patients, we compared the activity of wild-type (c.617G; R206) and mutant (c.617A; R206H) ACVR1 expression constructs on expression of the BMP pathway transcriptional target gene inhibitors of differentiation 1 (ID1) $(29,30)$. The wildtype construct contains arginine at codon 206 (R206), while the mutant construct replaces codon 206 with histidine $(\mathrm{R} 206 \mathrm{H})$. Cells were transfected with the human ID1 promoter $(-985 /+94)$ luciferase reporter construct (31) together with the wild-type or mutant ACVR1 expression construct. In COS-7 cells expressing the R206H ACVR1 mutant, the ID1 promoter activity was enhanced $3.0 \pm 0.2$-fold compared with cells expressing wild-type ACVR1 (Figure 1A). Similar results were observed in C2C12 and U-2 OS cells (Figure 1A). Both wild-type ACVR1 and mutant ACVR1 showed increased ID1 promoter activity in response to BMP treatment; however, the relative activity levels between the mutant and wild-type responses were not significantly different in these assays (data not shown). These data indicate that although the ACVR1 R206H mutant remains responsive to BMP ligand, this mutation does not require BMP ligand in order to activate BMP pathway signaling and ID1 gene transcription.

To determine whether BMP-independent expression of BMP target genes by mutant ACVR1 is mediated through specific BMP signaling pathways, we examined activation of Smad1, -5 , and -8 , which transduce intracellular signals upon phosphorylation by BMP receptors. Following transfection of COS-7 cells with wildtype ACVR1 or mutant R206H ACVR1 expression constructs, total proteins were assayed by immunoblotting for phosphorylated Smad1/5/8. Mutant ACVR1 induced Smad1/5/8 phosphorylation in the absence of exogenous BMP ligand (Figure 1B), consistent with BMP-independent stimulation of ID1 promoter activity. No Smad phosphorylation was observed in protein extracts from cells expressing equal levels of the wild-type ACVR1 in the absence of 
A IP: FKBP1A
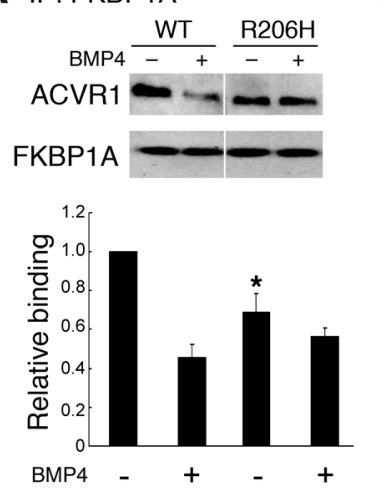

B IP: ACVR1
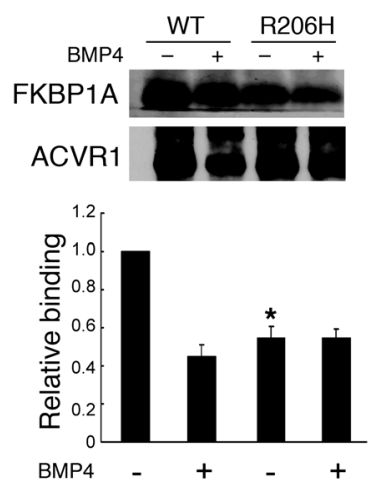

\section{Figure 2}

Reduced binding of FKBP12 to mutant ACVR1. COS-7 cells were cotransfected with FKBP1A expression vectors and wild-type ACVR1 or mutant ACVR1 (R206H) constructs. Following no treatment (-) or treatment $(+)$ with $150 \mathrm{ng} / \mathrm{ml} \mathrm{BMP4}$, proteins were $(\mathbf{A})$ immunoprecipitated with anti-FKBP1A/FKBP12 antibody, then immunoblotted with V5 antibody to detect V5-tagged ACVR1 or (B) immunoprecipitated with antiACVR1 antibody and immunoblotted to detect FKBP1A (top panels). The relative quantitative interactions between ACVR1 and FKBP12 are shown in the lower panels. Data represent mean \pm SEM. ${ }^{*} P<0.05$ versus wild-type without BMP treatment. The blots in lanes in $\mathbf{A}$ were run on the same gel but were noncontiguous (white line).

BMP ligand. Phosphorylation of Smad2, a Smad specifically activated by TGF- $\beta$ signaling, was not changed in response to mutant ACVR1, indicating specificity for the BMP signaling pathway (data not shown). Similar results were obtained using MC3T3-E1 (preosteoblast) cells (Figure 1B).

Independently of Smads, BMP signaling also activates the p38 MAPK pathway (32-34); however, additional assays in COS-7 cells showed no increase in p38 MAPK phosphorylation in response to ACVR1 (R206H) (data not shown). These data support the hypothesis that the BMP-independent activation of BMP signaling by the mutant R206H ACVR1 receptor is mediated, at least in part, through the canonical BMP Smad signaling pathway.

Mutant R206H ACVR1 shows impaired binding of FKBP1A in the absence of BMP ligand. To explore the mechanism for BMP-independent activation of the BMP pathway by the ACVR1 GS domain $\mathrm{R} 206 \mathrm{H}$ mutation, we examined the interaction of this receptor with the inhibitory protein FKBP1A/FKBP12, which binds to the GS domain of type I receptors and prevents leaky signal transduction in the absence of ligand binding (20-23). COS-7 cells were cotransfected with an FKBP1A expression construct and with wild-type or R206H mutant ACVR1 expression constructs. Immunoprecipitation of FKBP1A and subsequent immunoblotting to detect associated ACVR1 showed decreased interaction of FKBP1A with the ACVR1 mutant, compared with wild-type, in the absence of BMP (Figure 2A). Furthermore, while wild-type ACVR1 responded to exogenous BMP as expected by releasing ACVR1FKBP1A binding, little change was observed in the level of interaction between FKBP1A and the mutant ACVR1 receptor following BMP treatment (Figure 2A). Reciprocal immunoprecipitation experiments (IP for ACVR1, immunoblot for FKBP1A) showed consistent results (Figure $2 \mathrm{~B}$ ). These data suggest that altered GS domain conformation and/or GS domain phosphorylation that may affect either direct or indirect binding of FKBP1A to mutant ACVR1 are possible mechanisms for the BMP-independent activation of the downstream signals by the FOP mutant receptor.

The R206H ACVR1 mutation induces BMP-independent byperactivity of BMP signaling in vivo. Gradients of BMP signaling in zebrafish embryos direct dorsal-ventral cell fate specification during embryogenesis. Low levels of BMPs allow dorsal cell fate specification (e.g., neural tissue; formation of head structures), whereas higher levels of BMPs direct ventral cell fate specification (e.g., epidermis, blood, formation of tail structures). In zebrafish, Alk8, the ortholog of human ACVR1/ALK2, is a BMP type I receptor that mediates dorsal-ventral axial patterning in the embryo. In the zygotic alk8 mutant lost-a-fin (laf), BMP signaling is strongly reduced or absent in post-gastrula stages, causing an expansion of dorsal cell fates at the expense of ventral cell fates that results in a dorsalized phenotype with defects restricted to the tail (Figure 3, $A$ and B, and refs. 27, 28, 35). Microinjection of wild-type human ACVR1 RNA into alk $8^{-/-}$zebrafish embryos rescued approximately $80 \%$ of the injected embryos completely or partially (Figure 3, C and F), showing that human ACVR1 can function as a BMP type I receptor in this zebrafish model and substitute for Alk8.

By contrast, microinjection of mutant ACVR1 RNA (encoding $\mathrm{R} 206 \mathrm{H}$ ) into alk $8^{-/-}$zebrafish embryos induced a strongly ventralized phenotype at 1 day after fertilization (Figure 3, D-F), indicating that the FOP ACVR1 mutation induces hyperactivity of BMP signaling in the zebrafish embryo. Compared with the alk8 mutants rescued with wild-type ACVR1, R206H ACVR1-injected embryos displayed defects in both head and tail tissues (Figure 3), evidence that development was affected during gastrulation (35). This is further supported by the greatly expanded expression of the ventral markers eve 1 and gata 2 and the more restricted expression of the dorsal marker foxb1 during gastrulation (Figure 3, G-L).

As further evidence of BMP signaling activation, proteins isolated from zebrafish embryos were examined by immunoblotting for phosphorylated Smad1/5. (There is no known Smad8 in zebrafish.) Embryos that expressed the mutant R206H ACVR1 showed increased levels of $S \mathrm{mad} 1 / 5$ phosphorylation relative to embryos expressing the control ACVR1 (Figure 3M).

Mutant ACVR1 signaling during zebrafish embryogenesis is mediated through Smads. Injection of mutant R206H ACVR1 mRNA into wildtype embryos induced a range of ventralized phenotypes (Figure 4, A-D). To examine dependence of mutant ACVR1-induced ventralization on downstream signal transduction by Smad5, smad5 morpholinos, which cause strongly dorsalized (C4 and C5) phenotypes in wild-type embryos (36), were used to inhibit Smad5 expression. Similar to the effect on wild-type embryos (Figure 4, E and F), inhibition of Smad5 induced a dorsalized phenotype in 4-somite-stage embryos (Figure 4, G and H) showing a complete block of the ventralizing effect of the R206H ACVR1 mutant and indicating that Smad5 is required downstream of the mutant ACVR1 receptor to activate BMP signaling in zebrafish. These experiments demonstrate that robust activation of BMP signaling by the mutant R206H ACVR1 receptor requires the Smad pathway in vivo.

Additional evidence that the R206H ACVR1 mutant receptor activates BMP signaling through the canonical Smad pathway was provided by treatment with dorsomorphin (DM), a small mol- 


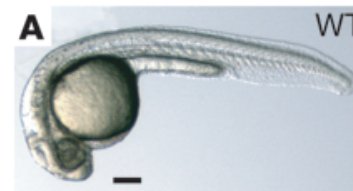

B
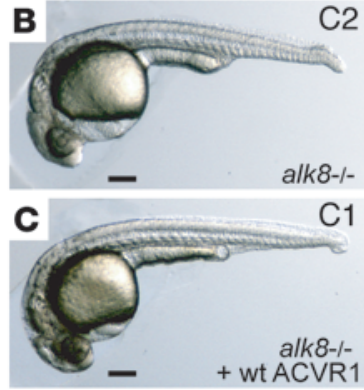

D

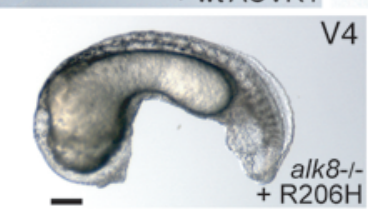

V4 E

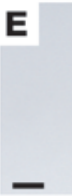

$\triangle$

$\Lambda$

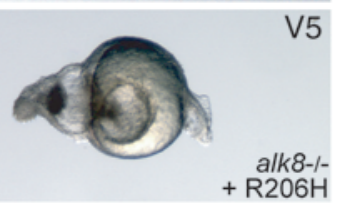

$\mathbf{F}$

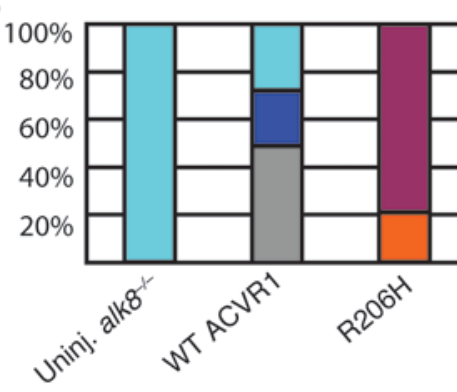

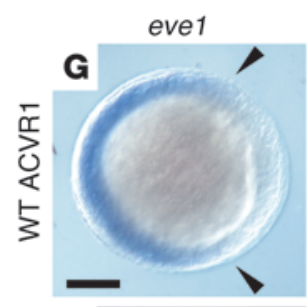
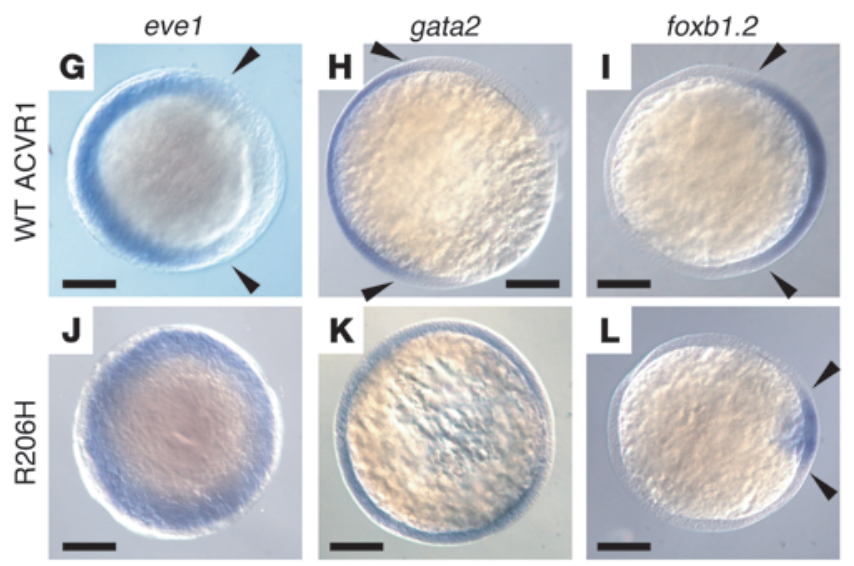

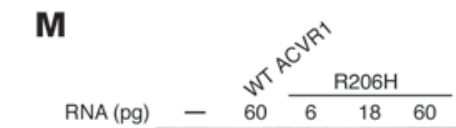

pSmad1/5

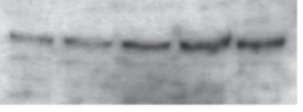

$\beta$-Actin

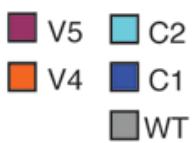

WT

\section{Figure 3}

Overexpression of R206H ACVR1 in zebrafish embryos causes strong ventralization by enhancing BMP signaling. Embryos from crosses between alk $8^{+/}$heterozygotes were injected with wild-type or mutant $(\mathrm{R} 206 \mathrm{H})$ ACVR1 mRNA, grouped by phenotype, then genotyped for the alk8 mutation. Compared with wild-type embryos (A), uninjected alk $8^{-1-}$ embryos are weakly (class 2; C2) dorsalized (B), with loss of the ventral fin fold (arrowheads). Wild-type ACVR1 mRNA (6-60 pg) at the 1-cell stage fully (as in A) or partially rescued the mutant phenotype (C1 dorsalized; C). (A-C) Embryos at 1 day after fertilization. Right panels show posterior of same embryos at 2 days after fertilization. (D and $\mathbf{E}$ ) Injection of $50 \mathrm{pg}$ mutant ACVR1 mRNA strongly (D) or severely (E) ventralized alk8 ${ }^{-/-}$embryos. Scale bars: $0.2 \mathrm{~mm}$. (F) Phenotype quantification of injected alk8-/- embryos (WT ACVR1, $n=43$; R206H, $n=15)$. (G-L) In situ hybridization of wild-type embryos injected with wild-type (G-I) or mutant ACVR1 (J-L) to detect ventral markers eve1 (G, $n=12 / 12 ; \mathbf{J}, n=10 / 13$; onset of gastrulation) and gata2 (H, $n=14 / 14 ; \mathbf{K}, n=15 / 19$; mid-gastrulation) or dorsal marker foxb1.2 (I, $n=12 / 12 ; \mathbf{L}, n=6 / 13,7 / 13$ showed no expression; mid-gastrulation). Animal pole views with dorsal at right. Arrowheads delineate the dorsal-ventral expression domains. Scale bars: $0.2 \mathrm{~mm}$. (M) Total mid-gastrulation stage protein from wildtype zebrafish embryos injected with wild-type or mutant ACVR1 mRNA was immunoblotted to detect phospho-Smad1/5. Increased Smad1/5 phosphorylation was observed even at a low dose $(6 \mathrm{pg})$ of mutant ACVR1. $\beta$-Actin was detected as a loading control.

ecule that inhibits BMP type I receptor signaling (ACVR1/ALK2, BMPR1A/ALK3, BMPR1B/ALK6) by specifically blocking Smad pathway activation (37). The reduced BMP signaling caused by DM induces severely dorsalized embryonic phenotypes in the zebrafish (Figure 4I) (37). To determine whether DM can disrupt the ventralizing activity of the R206H ACVR1 mutation, we injected wildtype embryos with mutant ACVR1 mRNA and treated them with $\mathrm{DM}$ or carrier solvent only (DMSO). As with the response of uninjected embryos, the majority of R206H-ACVR1-injected embryos that were treated with DM developed a class 5 severely dorsalized phenotype (Figure 4I). Inhibition of the mutant R206H ACVR1 receptor by DM is consistent with Smad-dependent signaling in the embryo and indicates that the mutant receptor requires the
Smad signaling pathway to activate BMP signaling and influence zebrafish development.

Mutant R206H ACVR1 effects on dorsal-ventral patterning are BMP independent. To complement our in vitro studies showing BMPindependent activation of BMP signaling, we investigated the function of the R206H ACVR1 mutant receptor in vivo by using zebrafish embryos lacking bmp2b and/or bmp7, the BMP ligands responsible for zebrafish dorsal-ventral patterning. Both $b m p 7$ and $b m p 2 b$ are required independently and nonredundantly for BMP signaling in the early zebrafish embryo due to the function of these ligands in a Bmp2b-Bmp7 heterodimer $(38,39)$. Thus, loss of either of these ligands causes loss of all BMP signaling in the early embryo and a C5 dorsalized phenotype that is identical to the 

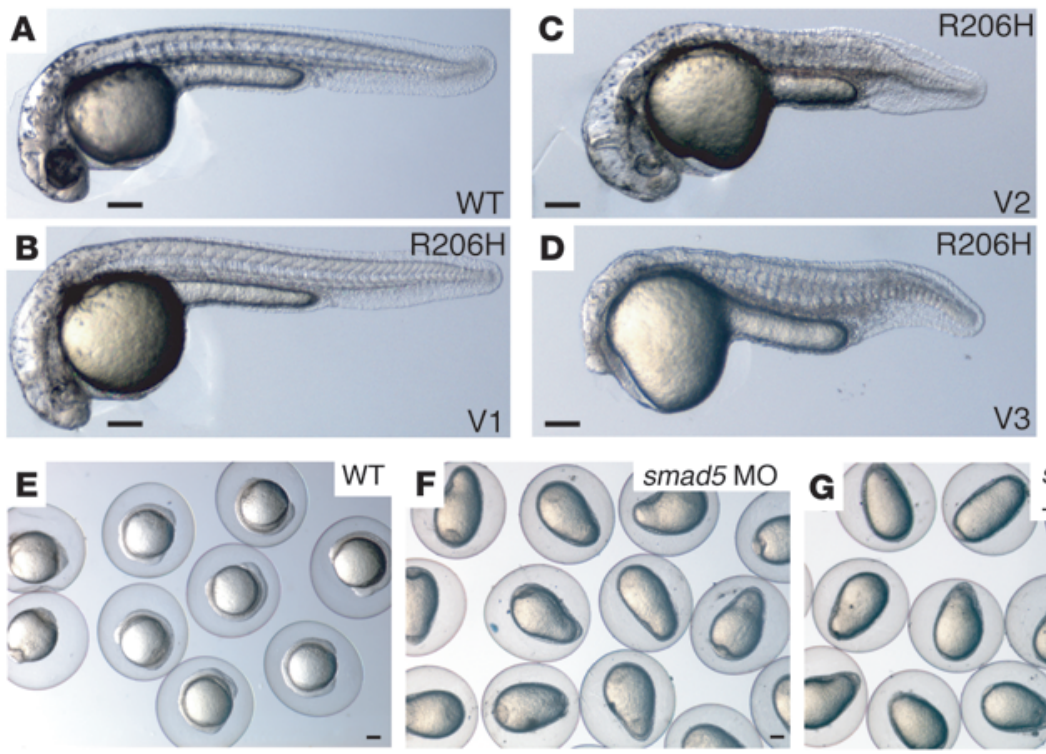

H

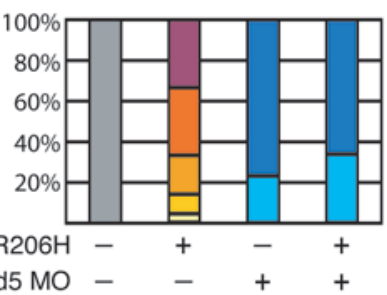

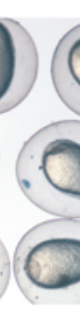

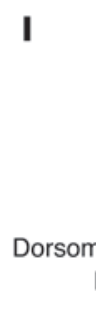

smad5 $\mathrm{MO}$
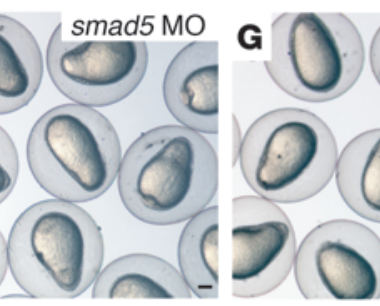

3
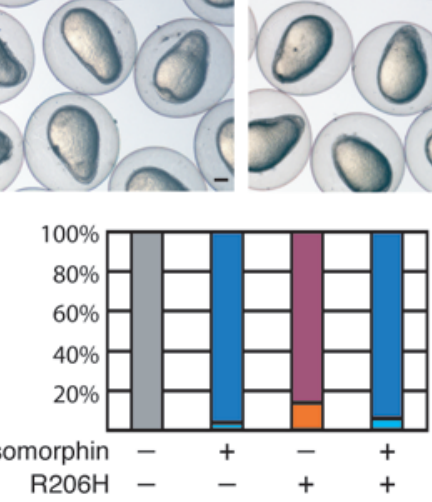

Figure 4

ACVR1 mutation signaling requires the BMP/Smad signaling pathway. (A-D) Compared with uninjected embryos (A), wild-type embryos injected with $10 \mathrm{pg}$ mutant R206H ACVR1 mRNA showed a range of ventralized phenotypes at 1 day after fertilization: weak (V1, B; and V2, C), moderate (V3, D), and strong (V4 and V5, examples shown in Figure 3, D and E). Scale bars: $0.2 \mathrm{~mm}$. (E-H) Compared with uninjected embryos (E), wild-type embryos injected with translation-blocking morpholinos (MO) against smad5 (F) showed strongly dorsalized phenotypes, evident by the characteristic elongated morphology at the 4-somite stage. When coinjected with smad5 $\mathrm{MO}$, mutant ACVR1 (10 pg) does not induce ventralization $(\mathbf{G})$. Scale bars: $0.2 \mathrm{~mm}$. $(\mathbf{H})$ Quantification of embryo injection results (uninjected, $n=82$; mutant ACVR1 only, $n=289$; smad5 MO only, $n=169$; mutant ACVR1 plus smad5 MO, $n=202$ ). (I) DM inhibits mutant $A C V R 1-i n d u c e d$ ventralization. Wild-type embryos treated with DM $(40 \mu \mathrm{M})$ become severely dorsalized $(n=54)$, whereas solvent alone has no effect $(n=42)$. Ventralization induced by 20 pg mutant ACVR1 mRNA $(n=79)$ is completely blocked by DM $(n=75)$. double $b m p 2 b / b m p 7$ mutant phenotype (39). Homozygous mutant bmp $2 b$ or $b m p 7$ embryos exhibited a strongly dorsalized phenotype (25\% of all progeny were C5), whereas sibling embryos (wild-type and heterozygotes; $75 \%$ of all progeny) showed a wild-type phenotype (Figure 5A). Microinjection of mutant R206H ACVR1 RNA induced embryonic ventralization, indicating BMP signaling overactivation, in the presence of BMP (wild-type or heterozygotes, Figure 5A), as well as in $b m p 7$ - or $b m p 2 b$-null embryos (Figure 5A). To completely knock down all BMP ligand expression in the embryos, we injected a $b m p 2 b$ morpholino into $b m p 7^{-1-}$ embryos. The single and double BMP ligand-deficient embryos (Figure 5B) showed strongly dorsalized phenotypes due to loss of BMP signaling. By contrast, single and double BMP-deficient embryos responded to injected mutant R206H ACVR1 by exhibiting a strongly ventralized phenotype (Figure 5B). Importantly, the wild-type ACVR1 showed no rescuing ability when injected into $b m p 7$-null mutant embryos ( $n=290,60$ pg ACVR1 mRNA). Thus, these results show that the mutant ACVR1 acts downstream of BMP ligand and activates $\mathrm{BMP}$ signaling in vivo in a BMP-independent manner.

Mutant R206H ACVR1 enhances chondrogenesis. Heterotopic bone formation in FOP patients develops through an endochondral ossification pathway in which a fibroproliferative vascular stage is followed by chondrogenesis and then replacement of cartilage by bone $(4,40)$. To investigate whether mutant R206H ACVR1 can influence chondrogenesis, we examined effects on chondrocyte differentiation by the ACVR1 mutation in chick limb bud micromass cultures that were infected with retroviral constructs containing either wild-type or mutant ACVR1. Overexpression of wild-type ACVR1 had no effect on extracellular matrix accumulation, which was monitored by Alcian blue staining, or on ALP activity when compared with cultures infected with empty viral vector (Figure 6). This is consistent with effects of other BMP receptors, such as BMPR1B (41). By contrast, overexpression of the R206H ACVR1 mutant induced a steady increase in matrix accumulation and ALP activity that were clearly visible after 1 week in culture. This effect was milder than that caused by a previously described constitutively active ACVR1 (caACVR1; Q207D), which was designed based on studies using the TGF- $\beta$ receptor 1 (42). The dramatic increase in chondrogenic differentiation in response to the caACVR1 Q207D was already visible after 2 days in culture and saturated after 1 week in culture (Figure 6).

To determine whether the effect of R206H ACVR1 is BMP independent, we coexpressed the receptor constructs with a construct for the BMP antagonist Noggin that directly binds BMP ligands, blocking the ability of BMP to bind receptors (43). Noggin had a dominant effect on wild-type ACVR1, as shown by complete inhibition of chondrogenic differentiation, while Noggin had no effect on caACVR1 Q207D activity. However, Noggin-treated cultures expressing mutant R206H ACVR1 had reduced chondrogenic nodule formation and ALP activity (Figure 6), showing that the FOP R206H ACVR1 mutation has a milder self-activating effect than the Q207D variant but, like this constitutively active variant, seems to be, at least in part, BMP independent. Significantly, by contrast to caACVR1 Q207D, R206H ACVR1 is BMP responsive. These data support the hypothesis that while the FOP ACVR1 mutation is an activating mutation that promotes BMP-independent chondrogenesis, this activity may be additionally responsive to BMP ligands.

To investigate chondrogenic differentiation on a molecular level, we analyzed the relative expression levels of marker genes that are characteristic for distinct stages of chondrogenesis (Figure 6E). 
A

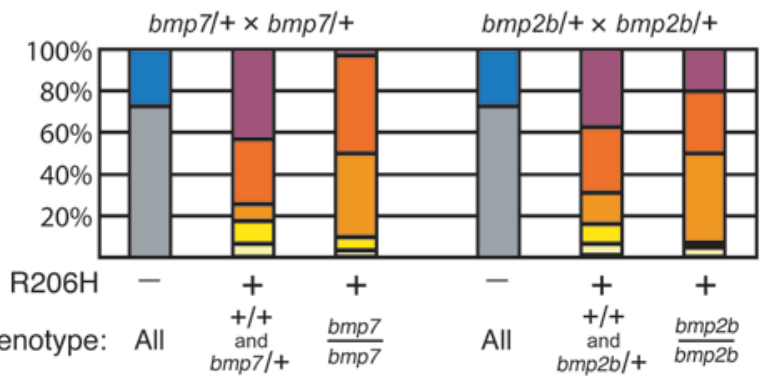

B

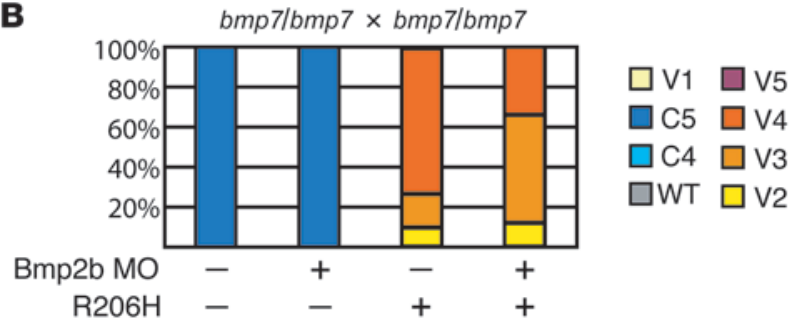

\section{Figure 5}

Mutant R206H ACVR1 induces ventralization in a BMP7 and BMP2b ligand-independent manner. (A) Embryos from crosses of bmp7 heterozygous adults (bmp7/ $\times$ bmp7/+) or bmp2b heterozygous adults $(\mathrm{bmp} 2 \mathrm{~b} /+\times \mathrm{bmp} 2 \mathrm{~b} /+$ ) were injected with $10 \mathrm{pg}$ mutant ACVR1 mRNA (indicated as R206H +), examined for extent of ventralization, then identified by genotyping (bmp7/bmp7, homozygous null for bmp7; bmp7/+, heterozygous; +/+, wild-type). Genotyping showed that bmp7 homozygotes $(n=32)$ and bmp2b homozygotes $(n=44)$ are ventralized by mutant $A C V R 1$, as are wild-type and heterozygous siblings (bmp $7 /+$ crosses, $n=94$; bmp $2 \mathrm{~b} /+$ crosses, $n=144$ ). Of the uninjected (indicated as R206 -) control embryos examined (for bmp7/+ $\times$ bmp $7 /+$ crosses, $n=146$; for bmp $2 b /+\times$ bmp2b/+ crosses, $n=29$ ), approximately one-quarter displayed a strongly dorsalized $C_{5}$ phenotype. (B) To examine whether mutant ACVR1 could activate BMP signaling in embryos lacking both Bmp7 and Bmp2b, embryos from crosses of bmp7 homozygous adults were injected with $10 \mathrm{pg}$ mutant ACVR1 mRNA with or without injection of a translation-blocking MO targeting bmp2b. Embryos exhibited a strongly dorsalized C5 phenotype in the absence of BMP ligands (uninjected, $n=34$; bmp2b MO, $n=34$ ), whereas mutant ACVR1 caused ventralization of embryos deficient in bmp7 $(n=126)$ or both bmp7 and bmp2b $(n=103)$.

The condensation phase of mesenchymal prechondrogenic cells is the first event in chondrogenesis, characterized by the expression of collagen type II (44). Aggrecan is a major component of cartilage matrix $(45,46)$ and is expressed in chondrocytes that are not terminally maturated (47). Within the condensation, increasing expression of Indian hedgehog (Ihh) and collagen type X occurs as chondrocytes differentiated into prehypertrophic and hypertrophic chondrocytes, respectively. Overexpression of wild-type ACVR1 in chick micromass cultures resulted in expression levels of marker genes that are comparable to those of the empty viral vector, while cells overexpressing R206H ACVR1 showed upregulation of collagen type II and aggrecan. The later-stage differentiation markers Ihh and collagen type $\mathrm{X}$ showed expression levels comparable to those in control cultures. By contrast, overexpression of caACVR1 Q207D resulted in a dramatic increase in Ihh and collagen type X expression. Aggrecan expression was only slightly enhanced, whereas collagen type II was markedly downregulated. Remarkably, the relative expression levels of all marker genes were only slightly decreased when R206H ACVR1 and Q207D caACVR1 cultures were cotransfected with Noggin, whereas cells expressing the wild-type ACVR1 or empty viral vector showed clear downregulation of all analyzed marker genes for chondrogenesis. These data support the hypothesis that while caACVR1 stimulates advanced chondrogenic differentiation, R206H ACVR1 only mildly induces the expression of early chondrogenesis markers.

\section{Discussion}

FOP is a severely disabling musculoskeletal disease characterized by extensive formation of endochondral bone within soft connective tissues. This heterotopic bone formation is the most clinically relevant feature of FOP. However, malformation of skeletal bones during embryogenesis also occurs in FOP patients and illustrates that the underlying genetic mutation occurs in a gene with functional importance in regulating chondro-osseous differentiation during embryonic skeletal development as well as in adult musculoskeletal tissues.

A series of recent investigations in FOP cells (48-50) demonstrated altered signal transduction through the BMP pathway, with increased phosphorylation of the BMP-specific Smads and increased expression of BMP transcriptional targets in the absence of exogenous BMP ligand. Our recent discovery (6) of mutations in the gene encoding ACVR1, a BMP type I receptor, in all examined FOP patients confirms that changes in the BMP signaling pathway cause FOP.

In this study, we conducted functional analyses of the ACVR1 c. $617 \mathrm{G} \rightarrow \mathrm{A}(\mathrm{R} 206 \mathrm{H})$ mutation that is found in all FOP patients with classic features of the disease (malformed great toes and progressive heterotopic ossification). Consistent with our studies in FOP patient cells (48-50), in vitro and in vivo assays determined that the R206H ACVR1 mutation is an activating mutation that stimulates BMP signaling without requiring BMP to initiate the signaling cascade. Our in vitro assays showed that BMP-independent BMP signaling by R206H ACVR1 specifically activates the Smad pathway, as was also found in recent studies using C2C12 cells (51). We further demonstrated that Smad signaling is required for $\mathrm{R} 206 \mathrm{H}$ ACVR1 signaling in vivo through zebrafish embryo assays using DM, a small molecule that inhibits BMP type I receptor phosphorylation of BMP pathway-specific Smads (37).

The FOP ACVR1 c.617A mutation causes an amino acid substitution of histidine for arginine in codon 206 (R206H), a highly conserved amino acid among vertebrates and among human ACVR1 family members (6). Codon 206 is within the GS activation domain adjacent to the protein kinase domain of ACVR1. Protein homology modeling of the ACVR1 receptor predicts that the protein conformation of the R206H ACVR1 mutant is altered and could lead to changes in the ability of the receptor to interact with proteins that bind the receptor GS domain $(6,52)$. The GS domain of all type I TGF- $\beta$ /BMP superfamily receptors is a critical site for binding and activation of the pathway-specific Smad signaling proteins and is a specific binding site for FKBP1A (also known as FKBP12), a highly conserved inhibitory protein that prevents leaky activation of type I receptors in the absence of ligand (21-23).

Our data demonstrate that the R206H ACVR1 protein has reduced interaction with FKBP1A in the absence of BMP and suggest that this impaired FKBP1A-ACVR1 interaction contributes to BMP-independent BMP pathway signaling. Although our data cannot distinguish between direct and indirect FKBP1A-ACVR1 binding, the data are consistent with the hypothesis that altered ACVR1 GS domain 
A

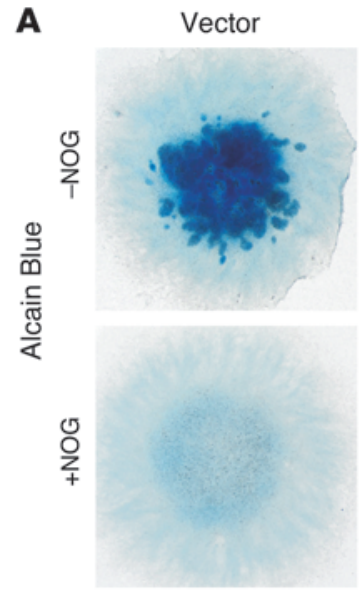

C

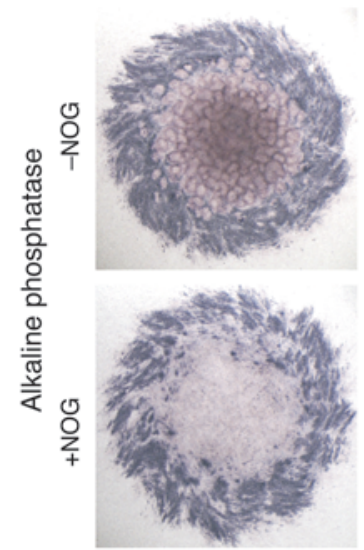

E

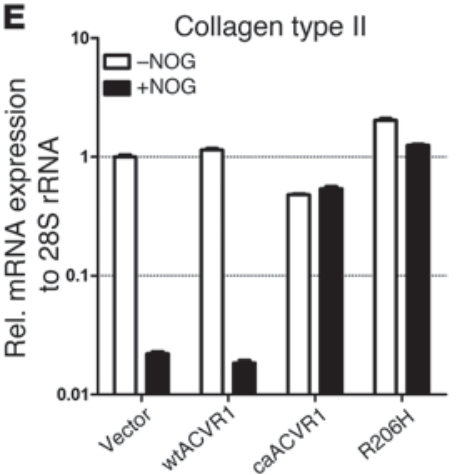

wtACVR1
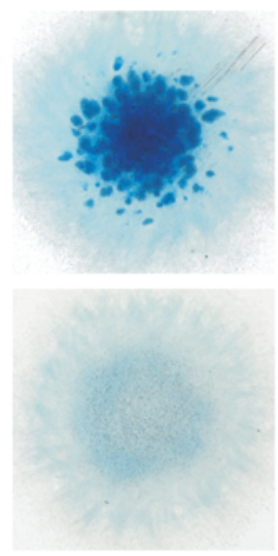

wtACVR1

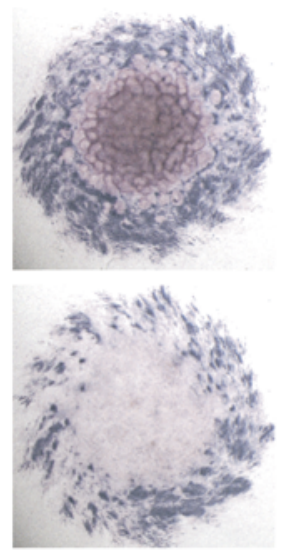

caACVR1
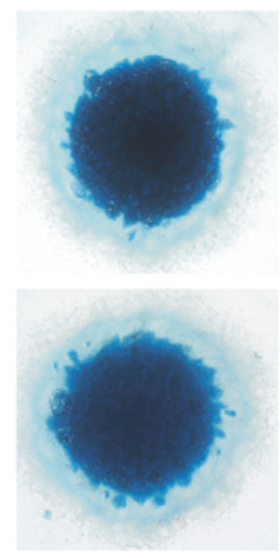

caACVR1
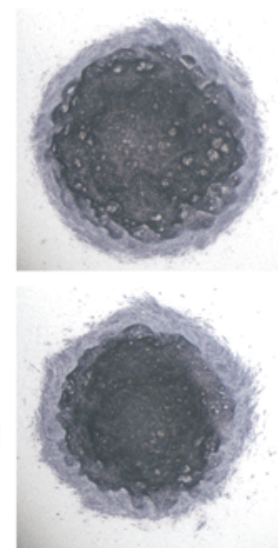

$\mathrm{R} 206 \mathrm{H}$

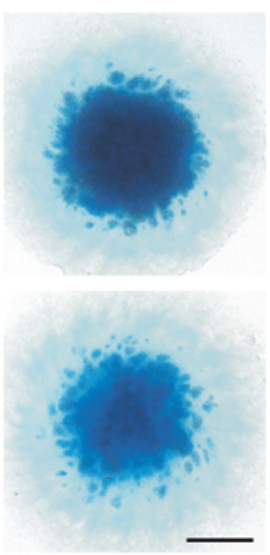

$\mathrm{R} 206 \mathrm{H}$
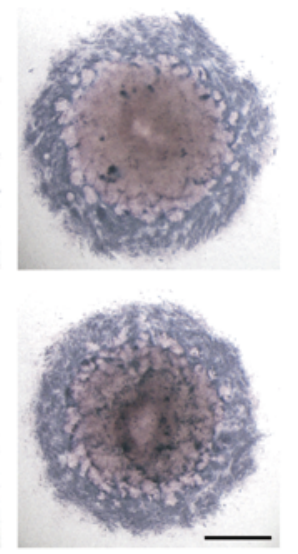

Ihh
B

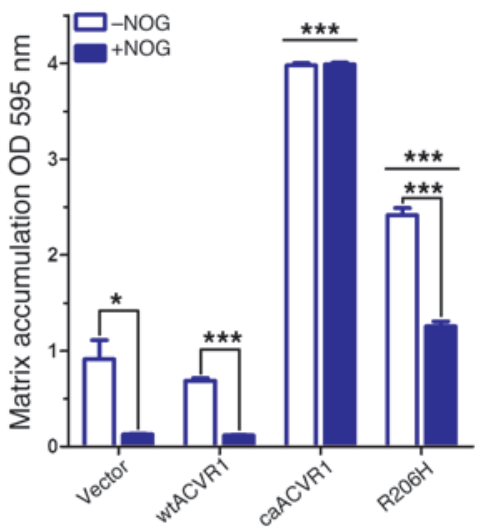

D

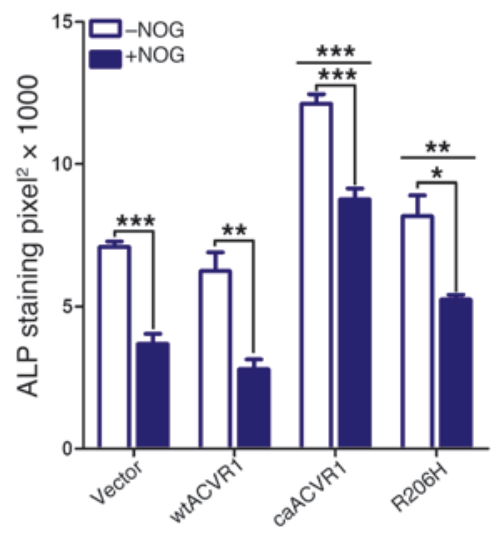

Figure 6

Mutant R206H ACVR1 enhances chondrogenesis in a BMP-independent and BMP-responsive manner. Micromass cultures were infected with $\operatorname{RCASBP}(\mathrm{A})$ alone (control) or containing either wild-type ACVR1, a constitutively active caACVR1 Q207D construct, or the R206H ACVR1 mutation either alone or together with RCASBP(B) expressing Noggin (NOG). (A) Micromass cultures were stained with Alcian blue to visualize early chondrogenic differentiation by extracellular matrix accumulation after 7 days. (B) Quantification of Alcian blue incorporation into the extracellular matrix is shown. Significant differences: wtACVR1 vs. caACVR1 ${ }^{\star \star *}$; wtACVR1+NOG vs. caACVR1+NOG***; wtACVR1 vs. R206H ${ }^{\star \star *}$; wtACVR1+NOG vs. R206H+NOG***. (C) ALP-stained micromass cultures visualize prehypertrophic differentiation after 9 days in culture. Scale bars in A and C: $2 \mathrm{~mm}$. (D) Quantification of ALP staining by histomorphometric analysis (ALP activity quantified as pixel2 $\times 10,000$ ) is shown. Significant differences: wtACVR1 vs. caACVR1 ${ }^{* *}$; wtACVR1+NOG vs. caACVR1+NOG***; wtACVR1 vs. R206H (NS); wtACVR1+NOG vs. $\mathrm{R} 206 \mathrm{H}+\mathrm{NOG}^{* *}$. (E) Relative expression of marker genes for chondrogenic differentiation by qRT-PCR of cDNA from 8-day micromass cultures. Amplification was normalized to the expression of 28S rRNA. For quantification in B and D, significant differences in comparison to wtACVR1 or wtACVR1+NOG are indicated. Graphs in B and D show mean \pm SEM of quadruplicate samples from 1 of 3 representative experiments. Horizontal lines represent significant differences at ${ }^{\star} P<0.05$; ${ }^{\star *} P<0.01$; ${ }^{* \star *} P<0.001$. Data in $\mathbf{E}$, shown as mean \pm SEM, are representative results from 2 independent cDNA syntheses and qRT-PCR. 
structure changes the affinity or ability of GS domain-interacting proteins, such as FKBP1A, to properly regulate BMP pathway activity. Additionally, our data show that the R206H ACVR1 mutation does not fully prevent FKBP1A interaction, suggesting that FKBP1A-mutant ACVR1 interactions are less stable and/or of shorter duration. The mutant GS domain may interact with FKBP1A in a manner that allows activation of Smads and downstream signaling in the presence of aberrant FKBP1A binding.

The BMP pathway is an important signaling pathway in many tissues and during many stages of development. Disruption of this pathway has severe consequences, as illustrated by knockout mouse models $(53,54)$ and by overactivation or by mutation of Alk8, the functional ACVR1 homolog in zebrafish $(7,27,28)$, and recent data demonstrated that Alk8 is required for zebrafish dorsal-ventral embryonic patterning (38). It was therefore surprising that the mutation that causes FOP overactivates the BMP pathway yet allows human embryonic development to occur relatively unimpaired, with only mild skeletal effects. One explanation may be that the FOP mutation is only moderately activating. By comparing FOP R206H ACVR1 with caACVR1 Q207D in micromass chondrogenesis assays, we found that the R206H ACVR1 receptor has a milder stimulation of cell differentiation compared with caACVR1 Q207D. The effects of the FOP ACVR1 mutant receptor during embryonic development may therefore induce only relatively minor malformations as observed in FOP skeletal formation $(1,6)$. Furthermore, the data are consistent with the idea that, in postnatal connective tissues, increased BMP signaling from the mutant receptor may be only moderately "on" under basal in vivo conditions, allowing for the quiescent periods that are observed in patients between active episodes of heterotopic bone formation, but priming the cells to respond to changes in the local tissue environment by forming extraskeletal bone.

This possibility is supported by micromass cultures expressing wild-type ACVR1, caACVR1 Q207D, or R206H ACVR1 that showed significant differences in expression of specific marker genes for chondrogenic differentiation. While expression levels of all marker genes remained almost unchanged in wild-type ACVR1 cultures compared with the empty viral vector, the expression profile induced by caACVR1 Q207D displayed evidence of advanced chondrogenic differentiation as shown by decreased levels of the early differentiation stage marker collagen type II together with enhanced expression of the later-stage markers Ihh and collagen type X. Indian hedgehog has been shown previously to be induced by BMP signaling transmitted via the ACVR1 receptor (9). By contrast, the R206H ACVR1 mutant only slightly enhances the expression of initial and early chondrogenesis markers (collagen type II and aggrecan), consistent with a milder stimulatory effect of the FOP ACVR1 mutation on chondrocyte differentiation. Remarkably, the BMP antagonist Noggin resulted in dramatically reduced expression of chondrogenic markers in wild-type ACVR1 and empty viral vector cultures, but not in those expressing caACVR1 or R206H ACVR1, suggesting that the enhanced chondrogenesis induced by the mutant receptors is mediated predominantly through their BMP-independent activation.

The extraskeletal bone that develops within soft tissues in FOP patients occurs through an endochondral ossification pathway, forming ectopic cartilage that is subsequently replaced by ectopic bone $(4,40)$. In vitro chondrogenesis assays demonstrated that the R206H ACVR1 mutation can stimulate progenitor cells to differentiate to cartilage in concert with the appropriate cell environment and differentiation factors and that this mutant receptor activates BMP signaling constitutively without BMP stimulation. However, chondrogenesis assays in chick micromass cultures showed that enhanced signaling by the mutant R206H ACVR1 can be partially inhibited by the BMP antagonist Noggin, suggesting that in addition to BMP-independent activity by the $\mathrm{R} 206 \mathrm{H}$ ACVR1 receptor, this mutant receptor is also responsive to BMP, consistent with previous BMP signaling pathway studies that used cells derived from FOP patients (48-50).

While our studies provide important insight into the molecular pathophysiology of the mutant ACVR1 receptor on the BMP signaling pathway in FOP, interpretations of these data are limited to those allowed by the experimental systems used. Most notably, the studies are based on transient transfection of wild-type and mutant gene constructs into cultured cells or into zebrafish embryos and are unlikely to reproduce the specific mutant gene dose that is present in heterozygous mutant cells in FOP patients. The balance of expression of mutant and wild-type ACVR1 protein is likely to affect ligand binding dynamics, receptor oligomerization, internalization, and/or activation of downstream signaling pathways, as well as receptor response to environmental triggers that are clinical features of the natural history of FOP. Further refinement of our understanding of BMP signaling in FOP will follow with development of an ACVR1 mutant knock-in mouse that will more precisely mimic the naturally occurring gene dose effect of the mutant R206H ACVR1 allele. Such a model is under development.

In conclusion, we show here that the recurrent single nucleotide missense mutation $(\mathrm{c} .617 \mathrm{G} \rightarrow \mathrm{A})$ in the gene encoding ACVR1, a BMP type I receptor that causes FOP in all classically affected individuals, induces enhanced chondro-osseous differentiation and can activate the BMP signaling pathway in a BMP-independent manner. However, this mutant receptor also shows the ability to respond to BMP to enhance receptor activity. Additionally, our study provides mechanistic insight into the constitutive activity of this mutant ACVR1 receptor. These findings have practical implications in developing model systems for testing new pharmacologic approaches for blocking this renegade receptor. Finally, this study provides insight into a signaling pathway that regulates skeletal morphogenesis and also that, when dysregulated in a specific manner, orchestrates the promiscuous and ectopic chondroosseous differentiation of soft connective tissue into a disabling second skeleton of heterotopic bone.

\section{Methods}

Plasmid constructs. A human ACVR1 expression vector was generated by insertion of the hACVR1 protein-coding sequence (GenBank accession number NM_001105.4) into the pcDNA 3.1 D V5-His-TOPO vector (Invitrogen). Nucleotide numbering reflects the ACVR1 cDNA sequence, with +1 corresponding to the A of the ATG translation initiation codon in the GenBank reference sequence, according to nomenclature guidelines (http://www.hgvs.org/mutnomen). The protein initiation codon is numbered +1 . The FOP mutant ACVR1 expression vector was generated by sitedirected mutagenesis of the wild-type ACVR1 sequence at CDNA position 617 (from G to A) using the GeneTailor Site-Directed Mutagenesis System (Invitrogen). The oligonucleotides used to generate the mutant construct were: forward 5'-GTACAAAGAACAGTGGCTCaCCAGATTACACTG-3'; reverse 5'-GTGAGCCACTGTTCTTTGTACCAGAAAAGGAAG-3'. The wild-type sequence is represented by "c.617G" and the FOP mutation by "c.617A." The FKBP1A/FKBP12 expression vector was from Origene. The human ID1 promoter $(-985 /+94)$ luciferase reporter construct was previ- 
ously described (31). The ID1 gene is a direct target of BMP signaling and encodes a dominant-negative inhibitor of basic helix-loop-helix transcription factors, including members of the MyoD family that are important in myoblast differentiation $(55,56)$.

Cell culture. COS-7 African green monkey kidney cells, C2C12 mouse myoblastic cells, MC3T3-E1 human osteoblastic cells, and U-2 OS human osteosarcoma cells were obtained from ATCC. Cells were cultured in DMEM (COS-7 and C2C12), $\alpha$-MEM (MC3T3-E1), or McCoy's 5A medium (U-2 OS) plus 10\% FBS (all from Invitrogen). All cells were cultured in a humidified atmosphere of $5 \% \mathrm{CO}_{2}$ at $37^{\circ} \mathrm{C}$.

Transfection and luciferase assay. COS-7 cells were seeded into 24-well plates at $7 \times 10^{4}$ cells per well in culture medium without antibiotics. After 24 hours, expression vectors were transfected into the cells using FuGene 6 (Roche) according to the manufacturer's protocol. Efficiency of transfection, as assessed by cotransfection with GFP constructs and subsequent GFP detection, was estimated at 60\%-70\%. At 48 hours, cells were washed twice with PBS and lysed in $1 \times$ passive lysis buffer (Dual-Luciferase Reporter Assay, Promega). Luciferase activity was assayed following the recommended protocol and normalized to pRL-TK-Renilla luciferase signals.

Immunoblot analysis. COS-7 cells, plated at $70 \%$ confluence in $100-\mathrm{mm}$ tissue culture dishes, were transfected with vector alone or pcDNA3-ACVR1 (c.617G) or pcDNA3-ACVR1 (c.617A). Total proteins were harvested in lysis buffer (20 mM Tris-HCl [pH 8.0], $150 \mathrm{mM} \mathrm{NaCl}$, phosphatase inhibitors [Pierce], protease inhibitors [C complete protease inhibitor cocktail, Roche], and $1 \%$ Triton $\mathrm{X}-100)$. For immunoblot analysis, $50 \mu \mathrm{g}$ protein from each total cell lysate was electrophoresed through $10 \%$ SDS-polyacrylamide gels, then transferred to PVDF membranes (Bio-Rad). Membranes were incubated overnight at $4{ }^{\circ} \mathrm{C}$ with antibodies specific for phospho-Smad1/5/8 and Smad1 (Cell Signaling Technology), V5 (Invitrogen), or $\beta$-actin (Santa Cruz Biotechnology Inc.) in PBS containing $5 \%$ nonfat milk and $0.5 \%$ BSA. Membranes were washed with PBS and incubated for 1 hour with the corresponding secondary antibody conjugated with horseradish peroxidase. The enhanced chemiluminescent Western blotting detection system (Pierce) was used to detect the antigen-antibody complex. Similar protocols were used for immunoblot analysis of cell protein extracts from MC3T3-E1 (mouse preosteoblasts), U-2 OS (human osteosarcoma), and C2C12 (mouse myoblasts with osteogenic potential). Western blotting of phospho-Smad1/5/8 from zebrafish embryo extracts was previously described (35).

Immunoprecipitation. To examine the interaction between FKBP1A and ACVR1 in the absence or presence of BMPs, COS-7 cells were cotransfected with normal (c.617G) or mutant (c.617A) ACVR1 expression vector and the FKBP1A expression vector. After 48 hours of transfection, cells were starved for 2 hours in serum-free medium then treated for 1.5 hours with $150 \mathrm{ng} / \mathrm{ml} \mathrm{BMP} 4$ or BMP7 (R\&D Systems). Total proteins were isolated, and protein concentration was detected by the Bradford assay. Immunoprecipitation assays used $500 \mu \mathrm{g}$ protein from each experimental sample and $2 \mu \mathrm{g}$ FKBP1A or ACVR1 antibody (both from Santa Cruz Biotechnology Inc.) at $4^{\circ} \mathrm{C}$ overnight, followed by treatment with $30 \mu \mathrm{l}$ of Protein $\mathrm{A} / \mathrm{G}$ agarose beads (Pierce) at $4^{\circ} \mathrm{C}$ for 1 hour and centrifugation at $800 \mathrm{~g}$ for 5 minutes. The immunoprecipitated complex was dissociated by $12 \%$ SDS-PAGE and detected with V5 monoclonal antibody (Invitrogen) or FKBP1A antibody (N19; Santa Cruz Biotechnology Inc.).

Micromass cultures. Viral constructs were designed and prepared for infection of chick limb bud mesenchymal stem cells in micromass culture. The coding sequence of chicken Acvr1 was PCR amplified from chicken embryo cDNA using the following primer pair: chAcvr1-NcoI-fwd, 5'-ACCATGGCTCTCCCCGTGCTGCTG-3' and chAcvr1-BamHI-rev, 5'-AGGATCCTCAACAGTCAGCCTTCAGTTT- $3^{\prime}$. The PCR product was digested with NcoI and BamHI and subcloned into the pSLAX-13 shuttle vector. This construct was used to introduce the corresponding human FOP mutation R206H and the constitutive active variant of the receptor Q207D by Site-Directed Mutagenesis (QuikChange, Stratagene) using the following primer pairs (with lower-case letters indicating the nucleotides changed relative to wildtype Acvr1 sequence): R206H-chAcvr1-fwd, 5'-GCAAAGAACAGTGGCTCaCCAGATCACGCTTGTGG-3' and R206H-chAcvr1-rev, 5'-CCACAAGCGTGATCTGGtGAGCCACTGTTCTTTGC-3'; chAcvr1-ca-Q207D-fwd, 5'-GCAAAGAACAGTGGCTCGCgAcATCACGCTTGTGGAGTG-3' and chAcvr1-ca-Q207D-rev, 5'-CACTCCACAAGCGTGATgTcGCGAGCCACTGTTCTTTGC-3'). Inserts were subcloned by $\mathrm{ClaI}$ into the avian-specific viral vector RCASBP(A). chNog in RCASBP(B) was a gift from A. Vortkamp (Universität Duisburg-Essen, Duisburg, Germany). To produce the virus, RCASBP plasmids were transfected into DF1 cells, culture medium was harvested, and viral particles were concentrated by ultracentrifugation as described previously (57). Titers of all receptor-expressing RCASBP(A) viruses were determined, and concentrations of greater than $1 \times 10^{8}$ were used throughout the experiments. The titer of Nog-expressing RCASBP(B) was $1 \times 10^{7}$.

Micromass cultures were prepared from dissected limb buds from chicken embryos at Hamburger-Hamilton stage HH24 as described previously (58). Cells $\left(2 \times 10^{5}\right)$ were plated in a drop containing $10 \mu$ l growth medium (DMEM:F12, 10\% FCS, $0.2 \%$ chicken serum, $2 \mathrm{mM} \mathrm{L-Gln}$, penicillin/streptomycin), and $1 \mu \mathrm{l}$ of each indicated viral concentrate was applied in a 24-well plate. Growth media were replaced 3 times a week. Alcian blue staining was performed after fixation of the cells in Kahle's fixative $(28.9 \%$ [v/v] EtOH; 0.37\% formaldehyde; 3.9\% [v/v] acetic acid) with $1 \%$ Alcian blue in $0.1 \mathrm{~N} \mathrm{HCl}$ overnight. Excess dye was removed by washing with water, and cultures were dried before photographs were taken. For quantification of incorporated Alcian blue into the proteoglycan-rich extracellular matrix, cultures were incubated with $6 \mathrm{M}$ guanidine hydrochloride overnight, followed by photometric measurement at $\mathrm{OD}_{595}$. ALP staining was performed after fixation of the cells with 4\% PFA and followed by incubation with NBT/BCIP. The reaction was stopped with TE buffer, and photographs were taken. For quantification, histomorphometric analyses were performed using Autmess AxioVision 4.6 software (Zeiss).

RNA isolation and relative quantitative RT-PCR. RNA was extracted from chicken micromass cultures at day 8 using peqGold Trifast (peqLab Biotechnologie $\mathrm{GmbH}$ ) following the manufacturer's instructions, then was digested with DNase (RNase-Free DNase kit, QIAGEN). cDNA synthesis was performed using the TaqMan Reverse Transcription Kit (Applied Biosystems). Quantitative RT-PCR (qRT-PCR) (ABIPrism 7900HT cycler, Applied Biosystems) was performed by using Power SYBR Green PCR Master Mix (Applied Biosystems) and the following primer pairs: ch-collagen type II-fwd, 5'-GAGGGCAACAGCAGGTTCAC-3' and ch-collagen type II-rev, 5'-TGCCCCATTTGCCAGTGT-3'; ch-collagen type X-fwd, 5'-CACTCTACTGCCTTGCATTGGA-3' and ch-collagen type X-rev, 5'AACAGCAGCAGTAACGATATTTGTAAA-3'; ch-Ihh-fwd, 5'-GGCTTTGACTGGGTCTACTACGA-3' and ch-Ihh-rev, 5'-CAGCCGAGTGCTCTGACTTG-3'; ch-aggrecan-fwd, 5'-TGTTTGTGTGTTTGCAAGCCA-3' and ch-aggrecan-rev, $5^{\prime}$-AGGCCATCGCTACTGTCTGAG-3'. To normalize gene expression, we used ch-28S rRNA-fwd, 5'-GGTATGGGCCCGACGCT$3^{\prime}$ and ch-28S rRNA-rev, 5'-CCGATGCCGACGCTCAT-3' (59) as a housekeeping gene. Relative expression levels normalized to 28S rRNA expression were calculated using qBase software (60).

Zebrafish embryo injection, plasmids, strains, and genotyping. PCR-amplified cDNA encoding control (c.617G) or mutant (c.617A) hACVRI was inserted into a derivative of $\mathrm{pCS} 2+$ vector (61) containing an in-frame C-terminal FLAG epitope (provided by M. Deardorff and P. Klein, University of Pennsylvania). mRNA was in vitro transcribed using the SP6 mMessage mMachine kit (Ambion) from plasmids linearized with NotI. Bmp7 and Bmp2b (ortholog of BMP2) are the BMPs that pattern the embryonic dorsal-ventral axis in zebrafish (14). A previously described morpholino against $b m p 2 b$ (62) 
was injected at $1 \mathrm{ng}$ per embryo. A morpholino mixture of $2 \mathrm{ng}$ smad5MO1 (5'-ATGGAGGTCATAGTGCTGGGCTGC-3') and $2.5 \mathrm{ng} \operatorname{smad} 5 \mathrm{MO} 3\left(5^{\prime}\right.$ GCAGTGTGCCAGGAAGATGATTATG-3') per embryo was used to knock down smad5 translation. All injections were performed at the 1-cell stage as described previously (63). Genotyping of the lost-a-fin ${ }^{t m 110 b}$ allele was performed as described (28). Homozygous null $b m p 7$ or $b m p 2 b$ mutant embryos were obtained from crosses between $b m p 7$ or $b m p 2 b$ heterozygous zebrafish. PCR genotyping for swirltdc24 (bmp2b) (64) used primers DC245P (5'-CGCTTGCTCAATATGTTCGGATTGAAT-3') and DC243P (5'-CGTGATGAAAACTTCGTATCGTGTTTG-3'), which create a recognition site for the restriction enzyme TaqI only when amplifying the wild-type allele. Homozygous $s n h^{\text {sblaub }}$ embryos ( $b m p 7$ ) (39) were identified by failure to PCR-amplify a closely linked simple sequence-length polymorphism (SSLP) using primers BMP7s42F (5'-CTCAGACACATAAGCCTAAGTGC-3') and BMP7s42R (5'-TCTATCCCAACTCAAATGCACCAG-3'). For treatment with DM, a BMP signaling inhibitor that dorsalizes zebrafish embryos (37), embryos were placed in E3 embryo medium containing DMSO either alone or with $40 \mu \mathrm{M}$ DM prior to first cleavage. All images of embryos were from an MZ12.5 stereomicroscope (Leica) with a ColorSNAP-cf digital camera (Photometrics) and processed using Adobe Photoshop. In situ hybridization and subsequent imaging were carried out as described previously (65) with probes against eve1 (66), gata2 (67), and foxb1.2 (68).

Statistics. Results are expressed as mean \pm SEM. A 2-tailed Student's $t$ test was used for statistical comparison of 2 groups. $P$ values of less than 0.05 were considered significant.

\section{Acknowledgments}

This work was supported in part by the Center for Research in FOP and Related Disorders, the International FOP Association, the Ian Cali Endowment, the Weldon Family Endowment, the Penn Center for Musculoskeletal Disorders, the Isaac and Rose Nassau Professorship of Orthopaedic Molecular Medicine, and the Deutsche Forschungsgemeinschaft (SFB760 to P. Seemann and S. Mundlos); by Health and Labour Science Research Grants for Research on Measures for Intractable Disease Research and the Academic Frontier Project of Saitma Medical University Research Center for Genomic Medicine, both from the Ministry of Education, Culture, Sports, Sciences, and Technology of Japan (to T. Katagiri); and by grants from the Rita Allen Foundation and the NIH (R01-GM056326 to M.C. Mullins; R01-AR40196 to F.S. Kaplan and E.M. Shore). We thank the members of our laboratories for valuable assistance and discussions of these studies.

Received for publication September 10, 2008, and accepted in revised form August 25, 2009.

Address correspondence to: Eileen M. Shore, University of Pennsylvania, Department of Orthopaedic Surgery, 424 Stemmler Hall, 3450 Hamilton Walk, Philadelphia, Pennsylvania 19104-6081, USA. Phone: (215) 898-2331; Fax: (215) 573-2133; E-mail: shore@ mail.med.upenn.edu.
1. Kaplan, F.S., et al. 2005. The phenotype of fibrodysplasia ossificans progressiva. Clin. Rev. Bone Miner. Metab. 3:183-188.

2. Cohen, R.B., et al. 1993. The natural history of heterotopic ossification in patients who have fibrodysplasia ossificans progressiva. A study of forty-four patients. J. Bone Joint Surg. Am. 75:215-219.

3. Connor, J.M., and Evans, D.A. 1982. Fibrodysplasia ossificans progressiva. The clinical features and natural history of 34 patients. J. Bone Joint Surg. Br. 64:76-83.

4. Glaser, D.L., et al. 2003. In vivo somatic cell gene transfer of an engineered noggin mutein prevents BMP4-induced heterotopic ossification. J. Bone Joint Surg. Am. 85-A:2332-2342.

5. Kaplan, F.S., et al. 1993. The histopathology of fibrodysplasia ossificans progressiva. An endochondral process. J. Bone Joint Surg. Am. 75:220-230.

6. Shore, E.M., et al. 2006. A recurrent mutation in the BMP type I receptor ACVR1 causes inherited and sporadic fibrodysplasia ossificans progressiva. Nat. Genet. 38:525-527.

7. Payne, T.L., Postlethwait, J.H., and Yelick, P.C. 2001. Functional characterization and genetic mapping of alk8. Mech. Dev. 100:275-289.

8. Tylzanowski, P., Verschueren, K., Huylebroeck, D., and Luyten, F.P. 2001. Smad-interacting protein 1 is a repressor of liver/bone/kidney alkaline phosphatase transcription in bone morphogenetic protein-induced osteogenic differentiation of C2C12 cells. J. Biol. Chem. 276:40001-40007.

9. Zhang, D., et al. 2003. ALK2 functions as a BMP type I receptor and induces Indian hedgehog in chondrocytes during skeletal development. J. Bone Miner. Res. 18:1593-1604.

10. ten Dijke, P., Korchynskyi, O., Valdimarsdottir, G., and Goumans, M.-J. 2003. Controlling cell fate by bone morphogenetic protein receptors. Mol. Cell. Endocrinol. 211:105-113.

11. Urist, M.R. 1965. Bone: formation by autoinduction. Science. 150:893-899.

12. Wozney, J.M., et al. 1988. Novel regulators of bone formation: molecular clones and activities. Science. 242:1528-1534.
13. Derynck, R., and Akhurst, R.J. 2007. Differentiation plasticity regulated by TGF-beta family proteins in development and disease. Nat. Cell Biol. 9:1000-1004

14. Little, S.C., and Mullins, M.C. 2006. Extracellular modulation of BMP activity in patterning the dorsoventral axis. Birth Defects Res. C. Embryo Today. 78:224-242.

15. Schmierer, B., and Hill, C.S. 2007. TGFbeta-SMAD signal transduction: molecular specificity and functional flexibility. Nat. Rev. Mol. Cell Biol. 8:970-982.

16. Harrison, C.A., et al. 2004. Modulation of activin and BMP signaling. Mol. Cell. Endocrinol. 225:19-24.

17. Lin, S.J., Lerch, T.F., Cook, R.W., Jardetzky, T.S., and Woodruff, T.K. 2006. The structural basis of TGFbeta, bone morphogenetic protein, and activin ligand binding. Reproduction. 132:179-190.

18. Miyazono, K., Maeda, S., and Imamura, T. 2005. BMP receptor signaling: transcriptional targets, regulation of signals, and signaling cross-talk. Cytokine Growth Factor Rev. 16:251-263.

19. David, L., Mallet, C., Mazerbourg, S., Feige, J.-J., and Bailly, S. 2007. Identification of BMP9 and BMP10 as functional activators of the orphan activin receptor-like kinase 1 (ALK1) in endothelial cells. Blood. 109:1953-1961.

20. Chen, Y.G., Liu, F., and Massague, J. 1997. Mechanism of TGFbeta receptor inhibition by FKBP12. EMBO J. 16:3866-3876.

21. Huse, M., Chen, Y.G., Massague, J., and Kuriyan, J. 1999. Crystal structure of the cytoplasmic domain of the type I TGF beta receptor in complex with FKBP12. Cell. 96:425-436.

22. Huse, M., et al. 2001. The TGF beta receptor activation process: an inhibitor- to substrate-binding switch. Mol. Cell. 8:671-682.

23. Yao, D., Dore, J.J., Jr., and Leof, E.B. 2000. FKBP12 is a negative regulator of transforming growth factor-beta receptor internalization. J. Biol. Chem. 275:13149-13154.

24. Shi, Y., and Massague, J. 2003. Mechanisms of TGFbeta signaling from cell membrane to the nucleus. Cell. 113:685-700.

25. Kawabata, M., Imamura, T., and Miyazono, K.
1998. Signal transduction by bone morphogenetic proteins. Cytokine Growth Factor Rev. 9:49-61.

26. Nohe, A., Keating, E., Knaus, P., and Petersen, N.O. 2004. Signal transduction of bone morphogenetic protein receptors. Cell. Signal. 16:291-299.

27. Bauer, H., Lele, Z., Rauch, G.J., Geisler, R., and Hammerschmidt, M. 2001. The type I serine/threonine kinase receptor Alk8/Lost-a-fin is required for $\mathrm{Bmp} 2 \mathrm{~b} / 7$ signal transduction during dorsoventral patterning of the zebrafish embryo. Development. 128:849-858.

28. Mintzer, K.A., et al. 2001. Lost-a-fin encodes a type I BMP receptor, Alk8, acting maternally and zygotically in dorsoventral pattern formation. Development. 128:859-869.

29. Korchynskyi, O., and ten Dijke, P. 2002. Identification and functional characterization of distinct critically important bone morphogenetic proteinspecific response elements in the Id 1 promoter. J. Biol. Chem. 277:4883-4891.

30. Maeda, Y., Tsuji, K., Nifuji, A., and Noda, M. 2004. Inhibitory helix-loop-helix transcription factors Id $1 /$ Id 3 promote bone formation in vivo. J. Cell. Biochem. 93:337-344.

31. Katagiri, T., et al. 2002. Identification of a BMPresponsive element in Id 1, the gene for inhibition of myogenesis. Genes Cells. 7:949-960.

32. Attisano, L., and Wrana, J.L. 2002. Signal transduction by the TGF-beta superfamily. Science. 296:1646-1647.

33. Derynck, R., and Zhang, Y.E. 2003. Smad-dependent and Smad-independent pathways in TGF-beta family signalling. Nature. 425:577-584.

34. von Bubnoff, A., and Cho, K.W. 2001. Intracellular BMP signaling regulation in vertebrates: pathway or network? Dev. Biol. 239:1-14.

35. Tucker, J.A., Mintzer, K.A., and Mullins, M.C. 2008. The BMP signaling gradient patterns dorsoventral tissues in a temporally progressive manner along the anteroposterior axis. Dev. Cell. 14:108-119.

36. Mullins, M.C., et al. 1996. Genes establishing dorsoventral pattern formation in the zebrafish embryo: the ventral specifying genes. Development. 123:81-93.

37. Yu, P.B., et al. 2008. Dorsomorphin inhibits BMP 
signals required for embryogenesis and iron metabolism. Nat. Chem. Biol. 4:33-41.

38. Little, S.C., and Mullins, M.C. 2009. BMP heterodimers assemble hetero-type I receptor complexes that pattern the DV axis. Nat. Cell Biol. 11:637-643.

39. Schmid, B., et al. 2000. Equivalent genetic roles for bmp7/snailhouse and bmp2b/swirl in dorsoventral pattern formation. Development. 127:957-967.

40. Gannon, F.H., et al. 1997. Bone morphogenetic protein $2 / 4$ in early fibromatous lesions of fibrodysplasia ossificans progressiva. Hum. Pathol. 28:339-343.

41. Lehmann, K., et al. 2003. Mutations in bone morphogenetic protein receptor $1 \mathrm{~B}$ cause brachydactyly type A2. Proc. Natl. Acad. Sci. U. S. A. 100:12277-12282.

42. Wieser, R., Wrana, J.L., and Massague, J. 1995. GS domain mutations that constitutively activate $\mathrm{T}$ beta R-I, the downstream signaling component in the TGF-beta receptor complex. EMBOJ. 14:2199-2208.

43. Groppe, J., et al. 2002. Structural basis of BMP signalling inhibition by the cystine knot protein Noggin. Nature. 420:636-642.

44. Kosher, R.A., Kulyk, W.M., and Gay, S.W. 1986. Collagen gene expression during limb cartilage differentiation. J. Cell Biol. 102:1151-1156.

45. Muir, H. 1995. The chondrocyte, architect of cartilage. Biomechanics, structure, function and molecular biology of cartilage matrix macromolecules. Bioessays. 17:1039-1048

46. Sekiya, I., et al. 2000. SOX9 enhances aggrecan gene promoter/enhancer activity and is up-regulated by retinoic acid in a cartilage-derived cell line, TC6. J. Biol. Chem. 275:10738-10744.

47. Han, Y., and Lefebvre, V. 2008. L-Sox5 and Sox6 drive expression of the aggrecan gene in cartilage by securing binding of Sox9 to a far-upstream enhancer. Mol. Cell. Biol. 28:4999-5013.

48. Billings, P.C., et al. 2008. Dysregulated BMP signaling and enhanced osteogenic differentiation of connective tissue progenitor cells from patients with fibrodysplasia ossificans progressiva (FOP). J. Bone Miner. Res. 23:305-313.

49. Serrano de la Pena, L.S., et al. 2005. Fibrodysplasia ossificans progressiva (FOP), a disorder of ectopic osteogenesis, misregulates cell surface expression and trafficking of BMPRIA. J. Bone Miner. Res. 20:1168-1176.

50. Fiori, J.L., Billings, P.C., Serrano de la Pena, L.S., Kaplan, F.S., and Shore, E.M. 2006. Dysregulation of the BMP-p38 MAPK signaling pathway in cells from patients with fibrodysplasia ossificans progressiva (FOP). J. Bone Miner. Res. 21:902-909.

51. Fukuda, T., et al. 2009. Constitutively activated ALK2 and increased SMAD1/5 cooperatively induce bone morphogenetic protein signaling in fibrodysplasia ossificans progressiva. J. Biol. Chem. 284:7149-7156

52. Groppe, J.C., Shore, E.M., and Kaplan, F.S. 2007. Functional modeling of the ACVR1 (R206H) mutation in FOP. Clin. Orthop. Relat. Res. 462:87-92.

53. Gu, Z., et al. 1999. The type I serine/threonine kinase receptor ActRIA (ALK2) is required for gastrulation of the mouse embryo. Development. 126:2551-2561.

54. Mishina, Y., Crombie, R., Bradley, A., and Behringer, R.R. 1999. Multiple roles for activin-like kinase-2 signaling during mouse embryogenesis. Dev. Biol. 213:314-326

55. Lopez-Rovira, T., Chalaux, E., Massague, J., Rosa, J.L., and Ventura, F. 2002. Direct binding of Smad1 and Smad 4 to two distinct motifs mediates bone morphogenetic protein-specific transcriptional activation of Id 1 gene. J. Biol. Chem. 277:3176-3185.

56. Melnikova, I.N., Bounpheng, M., Schatteman, G.C., Gilliam, D., and Christy, B.A. 1999. Differential biological activities of mammalian Id proteins in muscle cells. Exp. Cell Res. 247:94-104.

57. Logan, M., and Tabin, C. 1998. Targeted gene misexpression in chick limb buds using avian replica- tion-competent retroviruses. Methods. 14:407-420.

58. Seemann, P., et al. 2005. Activating and deactivating mutations in the receptor interaction site of GDF5 cause symphalangism or brachydactyly type A2. J. Clin. Invest. 115:2373-2381.

59. Li, T.-F., O'Keefe, R.J., and Chen, D. 2005. TGF-beta signaling in chondrocytes. Front. Biosci. 10:681-688.

60. Hellemans, J., Mortier, G., De Paepe, A., Speleman, F., and Vandesompele, J. 2007. qBase relative quantification framework and software for management and automated analysis of real-time quantitative PCR data. Genome Biol. 8:R19.

61. Rupp, R.A., Snider, L., and Weintraub, H. 1994. Xenopus embryos regulate the nuclear localization of XMyoD. Genes Dev. 8:1311-1323.

62. Imai, Y., et al. 2005. Hepatocyte growth factor contributes to fracture repair by upregulating the expression of BMP receptors. J. Bone Miner. Res. 20:1723-1730.

63. Westerfield, M. 1995. The zebrafish book. University of Oregon Press. Eugene, Oregon, USA. 385 pp.

64. Nguyen, V.H., et al. 2000. Dorsal and intermediate neuronal cell types of the spinal cord are established by a BMP signaling pathway. Development. 127:1209-1220.

65. Nguyen, V.H., et al. 1998. Ventral and lateral regions of the zebrafish gastrula, including the neural crest progenitors, are established by a bmp $2 \mathrm{~b} /$ swirl pathway of genes. Dev. Biol. 199:93-110.

66. Joly, J.S., Joly, C., Schulte-Merker, S., Boulekbache, H., and Condamine, H. 1993. The ventral and posterior expression of the zebrafish homeobox gene eve1 is perturbed in dorsalized and mutant embryos. Development. 119:1261-1275.

67. Detrich, H.W., 3rd, et al. 1995. Intraembryonic hematopoietic cell migration during vertebrate development. Proc. Natl. Acad. Sci. U. S. A. 92: 10713-10717.

68. Odenthal, J., and Nusslein-Volhard, C. 1998. Fork head domain genes in zebrafish. Dev. Genes Evol. 208:245-258. 